\title{
Nonlinear modelling of renal vasoaction
}

\author{
Violeta Mangourova ${ }^{\mathrm{a}, *}$, John Ringwood ${ }^{\mathrm{a}}$, Sarah-Jane Guild ${ }^{\mathrm{b}}$, Simon Malpas ${ }^{\mathrm{b}}$ \\ ${ }^{a}$ Department of Electronic Engineering, NUI Maynooth, Ireland \\ ${ }^{\mathrm{b}}$ Department of Physiology, University of Auckland, New Zealand \\ Received 28 February 2007; received in revised form 20 June 2007; accepted 4 July 2007 \\ Available online 17 August 2007
}

\begin{abstract}
The control of blood pressure is a complex mixture of neural, hormonal and intrinsic interactions at the level of the heart, kidney and blood vessels. While experimental approaches to understanding these interactions are useful, it remains difficult to conduct experiments to quantify these interactions as the number of parameters increases. Thus, modelling of such physiological systems can offer considerable assistance. Typical mathematical models which describe the ability of the blood vessels to change their diameter (vasoconstriction) assume linearity of operation. However, due to the interaction of multiple vasocontrictive and vasodilative effectors, there is a significant nonlinear response to the influence of neural factors, particularly at higher levels of nerve activity (often seen in subjects with high blood pressure) which leads to low blood flow rates. This paper proposes a number of nonlinear mathematical models for the relationship between neural influences (sympathetic nerve activity (SNA)) and renal blood flow, using a feedback path to model the predominantly nonlinear effect of local vasoactive modulators such as nitric oxide, which oppose the action of SNA. The model structures are motivated by basic physiological principles, while the model parameters are determined using numerical optimisation techniques using open-loop data collected from rabbits. The models were verified by demonstrating correlation between experimental results and model outputs.
\end{abstract}

(C) 2007 Elsevier Ltd. All rights reserved.

Keywords: Biomedical systems; Mathematical model; Blood flow; Nonlinear analysis; Numerical optimisation

\section{Introduction}

The regulation of blood pressure (BP) is critical in maintaining nutrient and oxygen supply to the various perfused organs. Blood pressure is determined according to the (Ohm's law) relationship:

$\mathrm{MAP}=\mathrm{CO} \cdot \mathrm{TPR}$

where MAP is the mean arterial pressure (measured in $\mathrm{mmHg}$ ), $\mathrm{CO}$ the cardiac output, evaluated as the product of heart rate and stroke volume (in $1 / \mathrm{s}$ ), and TPR is the total peripheral resistance as seen by the heart (in $\mathrm{mmHg} \mathrm{s} / \mathrm{l}$ ).

This study focuses on those components which mediate the resistance to blood flow (BF), while the heart is assumed to have a relatively constant output. This assumption is acceptable as the typical heart rate standard deviation is only about 1 beat/ min. $\mathrm{BF}$ is differentially regulated according to physiological

\footnotetext{
* Corresponding author.

E-mail address: violeta.i.mangourova@nuim.ie (V. Mangourova).
}

needs at any particular time via a variety of hormonal, neural and intrinsic factors. In particular, the emphasis is on TPR and how it mediates blood flow/pressure on a relatively short timescale, i.e. seconds. Central to this timescale, with a time delay between stimulation and response of $0.6 \mathrm{~s}$ [1], is the neural control of blood pressure, with sympathetic innervation of a number of major organs and areas of the vasculature, allowing rapid control of resistance via the central nervous system. Such sympathetic nerve activity (SNA) causes the release of neurotransmitters, which cause the smooth muscle surrounding small arteries and arterioles to constrict [2]. The distribution of sympathetic innervation throughout the vasculature determines the action that will take place at any particular site. However, in addition to neural control, several other mechanisms have significant effect on resistance, including:

- Hormones, which circulate throughout the system and can effect both vasodilation or vasoconstriction, depending on the particular hormone and the type of receptor it binds to (typical hormones include epinephrine, antidiuretic hormone, angiotensin II and cortisol $[3,4])$. 
- Intrinsic factors (myogenic autoregulation), which regulate blood vessel compliance and can produce a vasoconstrictive action in the smooth muscle in response to a distorting force on the walls of blood vessels due to BP [5].

- Paracrines, which are local humoral substances and can have both vasodilatory (e.g. prostacyclin, nitric oxide) and vasoconstrictive effects (e.g. thromboxane, endothelin-1 [6]).

- Metabolic factors, which can elicit vasoaction in response to local metabolic demands. Typical mediators include oxygen (constriction).

Fig. 1 (adapted from [7]) attempts to summarise the various factors involved in mediating vasoaction. The vasoactive mechanisms can be loosely grouped into systemic effects (e.g. SNA, hormones) and local effects (paracrines, tissue metabolites). The vasoaction at any particular site is therefore likely to be a combination of both factors. The study in this paper examines the renal vasculature in particular and attempts to build a mathematical model relating $\mathrm{BF}$ to SNA, with hormones, paracrines, etc., as mediating factors.

Mathematical models can be used to develop an understanding of the system under study. In order to achieve structural information, the emphasis should be on models which attempt to exploit the physical system description, rather than adopting a global black box modelling approach. While the latter approach can give a very good model fit for specific experimental data, it does little to reveal the generic structure of the system under study. A popular approach for modelling real systems, combining physical a priori knowledge of the system for model structure development, and numerical methods for parameter estimation is Gray Box modelling [8]. Once a model structure is defined, a number of numerical methods can be used for parameter optimisation. In [9] a number of optimisation techniques were compared to identify which optimisation method is mostly suited to the parameterisation of Model A described in the current paper.

To date, a number of attempts have been made to model the blood flow response to SNA. However, most techniques focus on linear models, which fail to capture essential aspects of the response. For example, the paper by Eppel et al. [10] considers

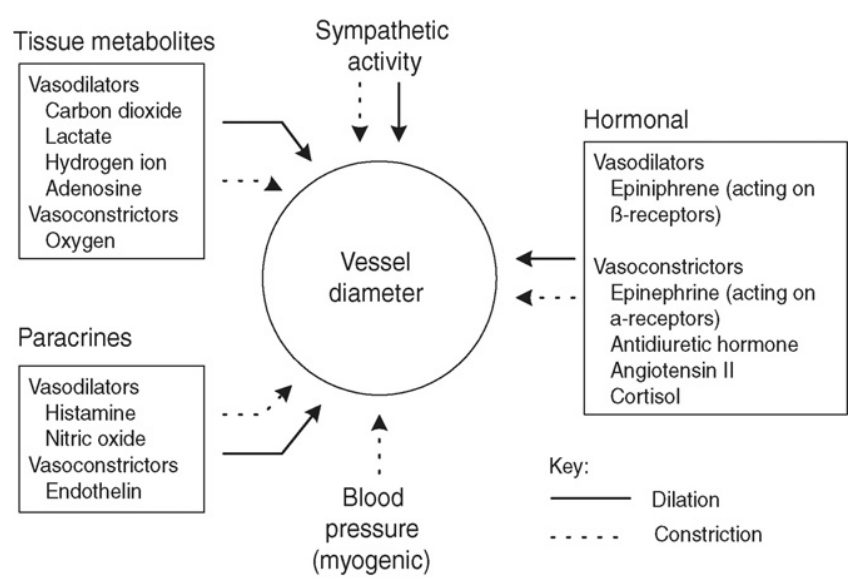

Fig. 1. Summary of vasoactive mechanisms. only broad magnitude changes in renal blood flow (RBF) in response to SNA stimulation, while the paper by Leonard et al. [11] fits an unparameterised frequency response to the RBF/ SNA relationship. Navakatikyan et al. [12] fit a first-order (pole-only) model to the response, which only models the RBF changes at low frequencies of stimulation, where the local vasodilatory factors are not visibly active. Guild et al. [1] use a frequency-domain approach to fit a linear fourth order (four poles and two zeros plus delay) model to the data. While this linear model gives a good fit at relatively low SNA amplitudes, the response match deteriorates at higher SNA amplitudes, as this higher stimulation level evokes reactionary responses in (local) vasodilatory mechanisms. Previous work by our group $[9,13]$ describe nonlinear mathematical models relating $\mathrm{RBF}$ to SNA. In [13] the model is only manually parameterised, while in [9] numerical optimisation was performed using various techniques and the resulting model fits were compared. Manual optimisation, gradient method (quasi-Newton algorithm), genetic algorithms (GAs) and a combination of GAs and the quasi-Newton algorithm were included in the comparison. Best results were obtained using a combination between GAs and the quasi-Newton method. The current study compares some of our previous work with a new model, comprising an improved model structure and parameters for three different animals.

The remainder of the paper is organised as follows: Section 2 deals with the various model structures proposed in this study, while available experimental data is presented in Section 3. Section 4 presents the optimisation techniques used and the results are outlined in Section 5. The conclusions and discussion are laid out in Section 6.

\section{Model development}

Three different models have been developed in this work and are presented in logical order, starting with the initial model accounted for in [13]. Structural and parametric improvements to this initial model are subsequently outlined.

The essential model structure is shown in Fig. 2. The neural control of the renal vasculature is considered central to the model for two reasons:

(1) It is the most significant (and 'independent') input to the model, i.e. most other mechanisms are considered reactionary on a more local level.

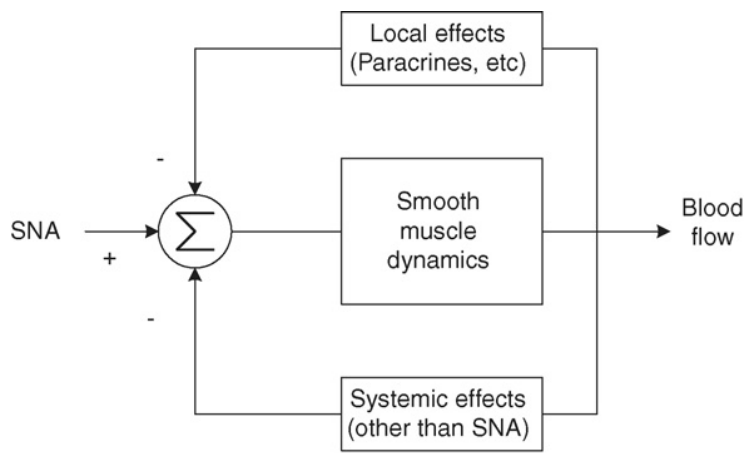

Fig. 2. Feedforward/feedback configuration. 
(2) The experimental data on which the model will be assessed is 'open-loop' as far as neural control of the vasculature is concerned. This is achieved via transection of the renal sympathetic nerve.

\subsection{Model structure A}

In Fig. 2, the two feedback paths represent the effects that both local and systemic blood pressure/flow control mechanisms have in response to SNA-based activation of RBF. Since the renal vasculature is just one component which regulates blood pressure, the response from systemic mechanisms is unlikely to be nearly as significant as the response from local mechanisms. Therefore, a single feedback block will be employed in the structure as shown in Fig. 3. This single block could incorporate systemic effects, but is configured to mainly model local effects. Model structure A is based on the following physiological premise. Above a certain (threshold) value of (normal) BF, the response of BF to SNA is relatively linear. However, when $\mathrm{BF}$ drops below a certain value, local factors work progressively harder (as $\mathrm{BF}$ decreases) to maintain an acceptable level of local BF. This response is captured by the 'activation level' block in Fig. 3. The 'local dynamics' block in Fig. 3 captures the speed of response of these local reactionary mechanisms. The 'smooth muscle dynamics' block represents the dynamic response of the smooth muscle to a stimulus from an appropriate receptor. Finally, a representation of the relationship between the frequency of SNA stimulation and steady-state BF response is added. This (mildly) nonlinear characteristic is given in Fig. 3 by the 'frequency' transform block.

Fig. 4 shows the typical type of response obtained from this model. The initial response to a step activation in SNA is roughly first-order exponential, but as soon as BF is reduced below a certain level, local (opposite) effects temper the response dramatically. Following release of the SNA activation, the response returns rapidly to the original level (and even overshoots it) assisted by the slowly dispersing local vasodilators. From this description, some aspects of the model can be clarified:

- The local vasodilatory response is not linear and has some 'threshold' of blood flow below which it is activated.

- The response of the local vasodilatory reaction is significantly slower than that of the smooth muscle to the SNA stimulus (i.e. $\tau_{\mathrm{b}}>\tau_{\mathrm{f}}$ ).

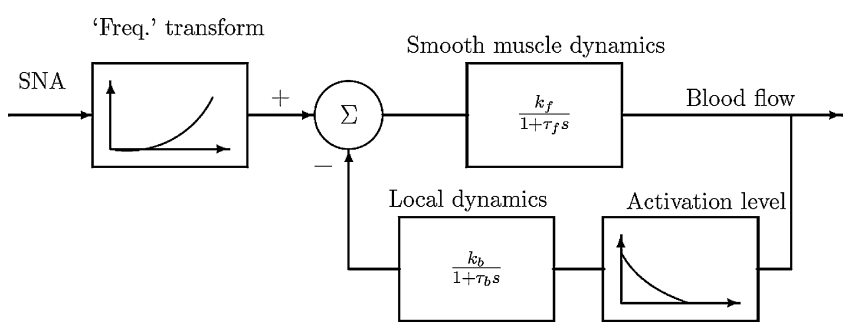

Fig. 3. Block diagram of Model A.

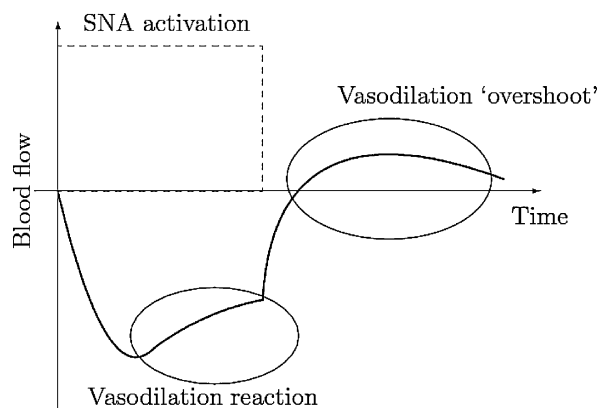

Fig. 4. Typical large-signal response to SNA activation.

- The magnitude of the action (to SNA) and reaction (by the local vasodilatory mechanism) is comparable, at least to an order of magnitude.

\subsection{Model structure $B$}

Model B, which represents an alternative structure, as shown in Fig. 5, employs the same blocks as Model A. However, in the case of Model B, the smooth muscle dynamics are taken outside of the feedback control loop. This structure represents the reduction in $\mathrm{BF}$ due to the neural constrictory effectors being counteracted by the increase in BF due to the local dilatory effectors. The sympathetic input to the smooth muscle dynamics block, however, is not affected by the output of the local dynamics block, as it is in Model A. This modification is justified since the smooth muscle response to SNA does not change with the addition of local vasodilatory factors.

\subsection{Stimulation/recovery models}

A further necessary enhancement of the model accounts for the different time constants during the stimulation and recovery stages of the experiment. The differences are due to different activation and dispersion rates, with the slower response during the recovery from stimulation. This is perfectly reasonable, since:

- Recovery from SNA activation is passive and the smooth muscle may take longer to relax than contract under forced activation and

- Hormones, paracrines, etc., may take much longer to disperse than the rate at which they were formed.

Thus, the model structure may remain the same for the two stages of the experiment, but the parameters need to be defined separately for stimulation (180 s) and recovery (300 s) in order

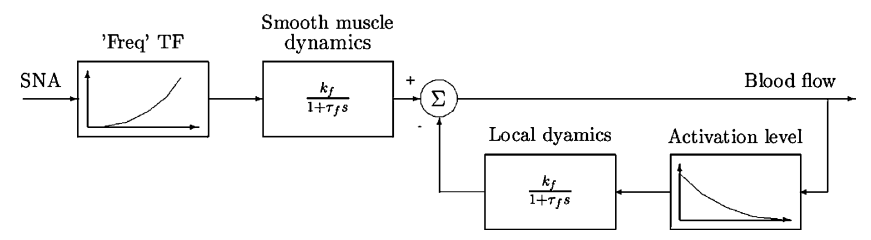

Fig. 5. Block diagram of Model B. 
to obtain a better representation of the real system. Considering its better portrayal of the underlying physiology, Model B was selected for separate numerical optimisation of stimulation and recovery.

\section{Data availability}

Experiments were performed on six anaesthetized New Zealand white rabbits [11]. A transit time flow probe (type 2SB; Transonic Systems, Ithaca, NY, USA), connected to a compatible flowmeter (T106, Transonic Systems) was used to measure RBF, with arterial pressure being monitored using a catheter inserted into the central ear artery and connected to a pressure transducer (Cobe, Arvarda, CO). The measured signals were sampled at $500 \mathrm{~Hz}$, digitized and saved continuously as $2 \mathrm{~s}$ averages of each variable. In addition, heart rate (HR, beats/min) was derived from the arterial pressure waveform.

For stimulation, the renal nerves were placed across a pair of hooked stimulating electrodes and then sectioned proximal to the electrodes. Stimulation sequences using frequency modulation (FM) were applied using a pulse width of $2 \mathrm{~ms}$. Frequencies of $1.5,2.0,3.0,5.0$ and $8.0 \mathrm{~Hz}$ were applied in random order using a voltage equal to that required to produce a maximal RBF response. The stimulation interval was $3 \mathrm{~min}$, with a 5 min recovery period.

\section{Model parameterisation}

In order to determine the best model parameters, an objective function $\left(J_{\text {tot }}\right)$ to be minimised was defined as the sum of the errors $\left(J_{k}\right)$ obtained for each frequency of stimulation ( $n=$ number of frequencies of stimulation used):

$$
J_{\text {tot }}=\sum_{k=1}^{n} J_{k}
$$

where $J_{k}$ is defined in the form of the mean squared error (MSE) as

$J_{k}=\frac{1}{N} \sum_{i=1}^{N}\left(y_{i}-\hat{y}_{i}\right)^{2}$

where $\hat{y}_{i}$ is the blood flow data obtained from the model simulations and $y_{i}$ is the actual blood flow data.

Normalised blood flow, representing the percentage change in blood flow from the initial blood flow level of each animal, was used for model development in this study. The normalisation was performed separately for each frequency of stimulation. Thus, the initial blood flow values correspond to $0 \%$ change in blood flow, while blood flow level of $0 \mathrm{mmHg}$ corresponds to $-100 \%$ change in blood flow. The intermediate blood flow levels then correspond to values between $0 \%$ and $-100 \%$ change during stimulation (due to reduction in blood flow) and can take on positive values as well during recovery (due to overshoot in blood flow). For reference purposes, the mean actual initial blood flow values for the three animals and for each frequency is $27.5857 \mathrm{mmHg}$ with a standard deviation of $5.76 \mathrm{mmHg}$.

\subsection{Manual parameterisation}

Given the intuitive nature of the initial model and the strong relationship with the underlying physiology, the first attempts focussed on tuning the model parameters by trial and error. There are four parameters in the dynamic blocks, as well as two piecewise linear functions, to determine. The relationship between SNA and blood flow is approximately linear above a certain threshold level of RBF, hence the feedforward loop dynamics are relatively easy to determine. However, below that threshold level, local factors are activated to oppose the reduction in $\mathrm{BF}$, thus activating the feedback path in the model. This latter relationship is nonlinear, hence more challenging to parameterise manually. Once the potential of the model was ascertained by relatively good results, more advanced numerical techniques were applied to the models, namely genetic algorithms and gradient techniques.

\subsection{Genetic algorithms and quasi-Newton method}

Genetic algorithms were employed to find a good MSE solution, as they are suitable for problems with irregular problem surfaces, where a global minimum may be difficult to find. GAs, however, do not guarantee to find the exact minimum, as their solution space is represented by discrete solution points. To overcome this problem and to find an exact minimum in the vicinity of the overall solution obtained by the GAs, a gradient technique can be employed. Gradient methods are often unable to find a global minimum in an irregular solution space, however they are suitable to finding exact local minima. Thus, a quasi-Newton algorithm was selected to determine the exact local minimum in the vicinity of the already obtained GA solution. Most Newton gradient search algorithms require calculations of the gradient and Hessian (second partial derivative, representing the curvature). However, numerical calculation of the Hessian can lead to loss of precision and even divergence from the minimum, in some cases. The quasiNewton method differs from similar Hessian-based techniques, in that the parameter approximations for the next step are calculated using estimates of the Hessian, calculated in a specific manner to reduce precision loss [14] and ensure convergence.

\section{Results}

Numerous sets of results are presented in this section. For each model, a table with the dynamic parameters and cost function will be shown, as well as two figures containing the piecewise linear functions. Results from the initial manual parameterisation of Model A, used to assess the potential of the model, are shown in Section 5.1. Next Models A and B were optimised using GAs and a gradient technique. These two sets of results are compared in Section 5.2 and represent data for a single animal. Model B, which has a better data fit, is then 
Table 1

Dynamic block parameters for manual parameterisation

\begin{tabular}{lc}
\hline$k_{\mathrm{f}}$ & 1 \\
$k_{\mathrm{b}}$ & 80 \\
$\tau_{\mathrm{f}}(\mathrm{s})$ & 20 \\
$\tau_{\mathrm{b}}(\mathrm{s})$ & 200 \\
$J_{\text {tot }}$ & 234.59 \\
\hline
\end{tabular}

optimised separately for the stimulation and recovery stages of the experiment and the results for three animals are presented in Section 5.3.

\subsection{Manual parameterisation}

The model parameter values and minimum cost obtained by Model A's manual optimisation are detailed in Table 1. All parameters were determined by trial and error to fit all frequencies of stimulation.

The two piecewise linear components in the model, namely the frequency transform function and the local vasodilators activation level, are shown in Figs. 7 and 8, respectively, and are marked as 'Manual'. A comparison of the model output and the experimental data for Rabbit 1 are shown in Fig. 6. Clearly, even with manual parameterisation, the model has captured the essence of the response contained in the experimental data, with the exception of the noise present in the physiological data. Arguably a better comparison could be made if the experimental data had been filtered prior to plotting, but the filter would introduce dynamics of its own, which may interfere with the parameter determination.

\subsection{Models $A$ and $B$ comparison}

The results for Model A and Model B, optimised with GAs and the quasi-Newton method, are compared in this section. The dynamic parameters, as well as the minimum cost (for Rabbit 1) are presented in Table 2. The models were trained

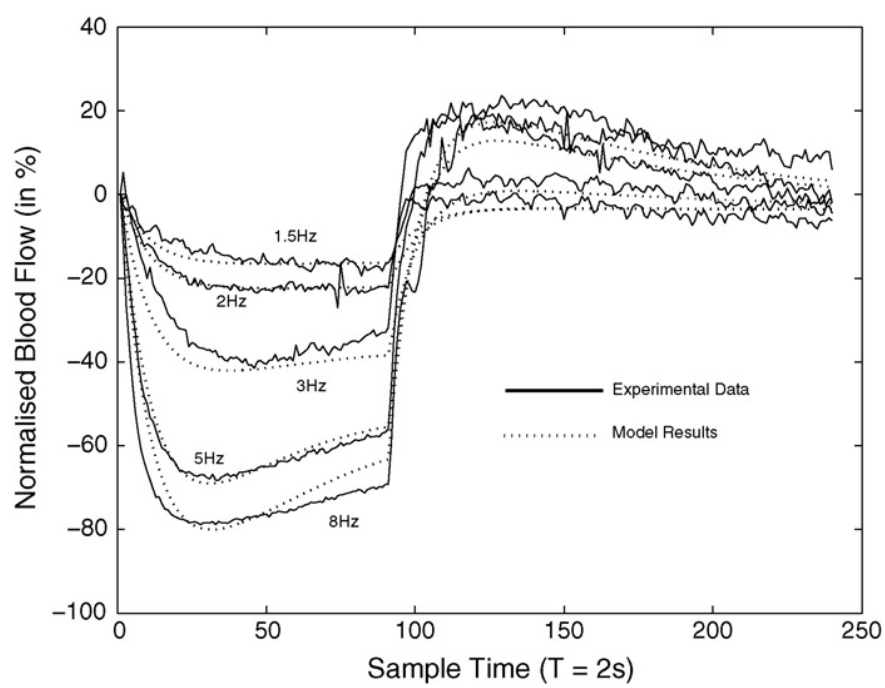

Fig. 6. Comparison of model response with experimental data for Model A (manually optimised parameters).
Table 2

Dynamic parameters for GAs and gradient techniques

\begin{tabular}{lllllr}
\hline Model & $k_{\mathrm{f}}$ & $k_{\mathrm{b}}$ & $\tau_{\mathrm{f}}(\mathrm{s})$ & $\tau_{\mathrm{b}}(\mathrm{s})$ & \multicolumn{1}{c}{$J_{\text {tot }}$} \\
\hline Model A & 1.04 & 72.15 & 20.09 & 179.98 & 102.46 \\
Model B & 1.12 & 83.0974 & 17.86 & 150.45 & 97.19 \\
\hline
\end{tabular}

with all frequencies of stimulation. The two piecewise linear functions are again shown in Figs. 7 and 8. The functions are marked as 'Model A' and 'Model B' on each of the figures. As shown in Table 2, Model B yielded marginally better results, thus it was selected for further enhancement, namely separate numerical optimisation of the stimulation and recovery stages of the model.

\subsection{Structure B stimulation/recovery models}

As previously outlined, the model structure yielding better results, Model B, is parameterised separately in this section for the stimulation and recovery stages of the experiment, thus accounting for the different $\mathrm{BF}$ responses during stimulation and relaxation. Again, GAs and quasi-Newton method were used. In the GA optimisation, the average population size used was 700 individuals. The starting values were randomly determined by the algorithm. Convergence on the final results, with a tolerance $<10^{-5}$ was reached on average after 57 iterations. In the case of the quasi-Newton method, the starting values used were the final results of the genetic algorithm for each individual animal. The quasi-Newton method provided minor improvements to converge on the local minima in the vicinity of the GA solutions in an average of 252 iterations.

The stimulation and recovery models, which have the same structure, are parameterised for three different animals. First, the models were optimised for Rabbit 1 , the original data set used for model structure development. Then the stimulation and recovery models were parameterised to fit two other animal sets

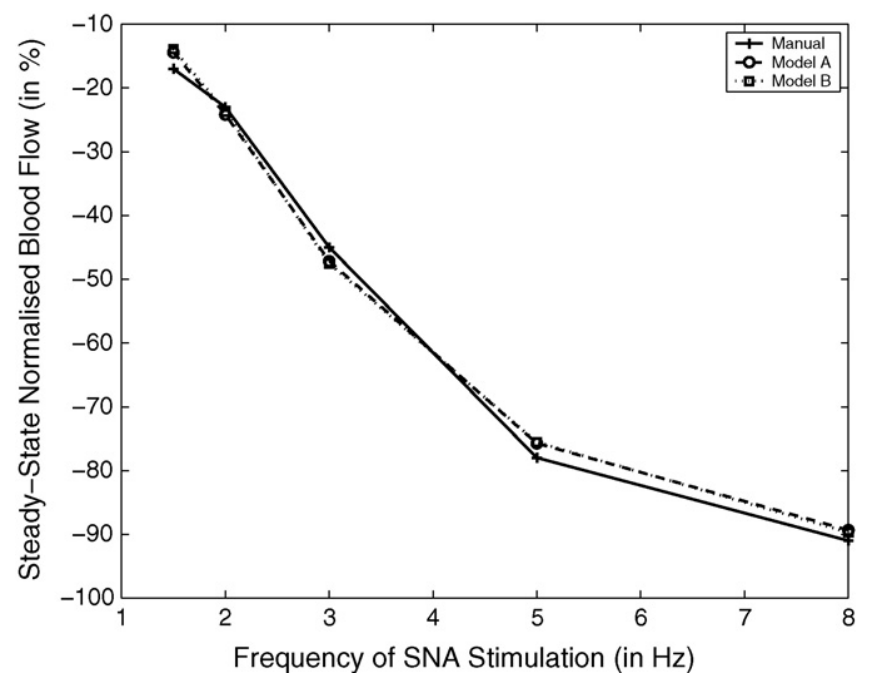

Fig. 7. 'Frequency' transform function for the manually optimised Model A (manual) and the computer optimised Models A and B. 


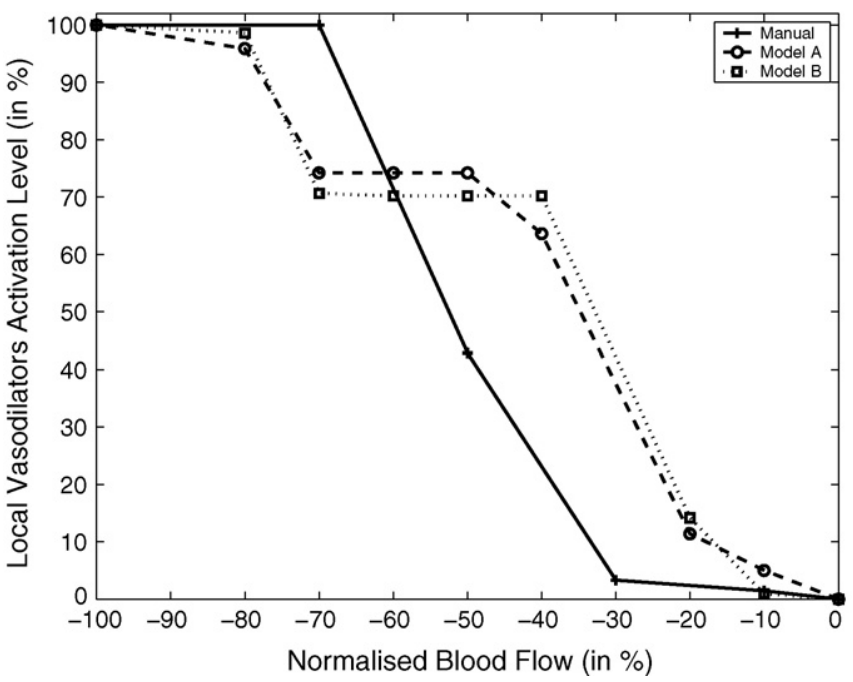

Fig. 8. Local vasodilators 'activation level' for the manually optimised Model A (manual) and the computer optimised Models A and B.

(Rabbits 2 and 3) to assess the quality of the model structure, when used across a number of animals. All results are presented in Table 3 and Figs. 9-11. The models are trained on four frequencies of stimulation, $1.5,2,5$ and $8 \mathrm{~Hz}$, and are tested with the fifth frequency of $3 \mathrm{~Hz}$, with $J_{\text {test }}$ being the MSE obtained for the test frequency.

Table 3 shows the significant differences between the dynamic parameters of the stimulation and recovery models, with larger gains and time constants during recovery, as expected. It is evident, from Table 3, that the stimulation models perform consistently better than the recovery ones for all three animals. In addition, Table 3 shows that Rabbits 2 and 3 are not as well represented by their models as the original data set (Rabbit 1), for which the model structure was built. The test results for $3 \mathrm{~Hz}$ are inferior to the training results. This, however, is misleading as if we were to include the $3 \mathrm{~Hz}$ data in the training data, the cost function for the $3 \mathrm{~Hz}$ set results in very similar values. This confirms the generalisation capability of the model and suggests that the $3 \mathrm{~Hz}$ data has some different properties to the rest of the data sets.

Fig. 9 shows the 'frequency' transform function for all three animals for the stimulation model. During recovery the model's input is zero, therefore this nonlinear block's output is zero for all frequencies of stimulation and does not require graphical representation. Similar values to ours, for normalised BF response to SNA stimulation frequencies of 1.5 and $2 \mathrm{~Hz}$, were reported by diBona and Sawin [15].

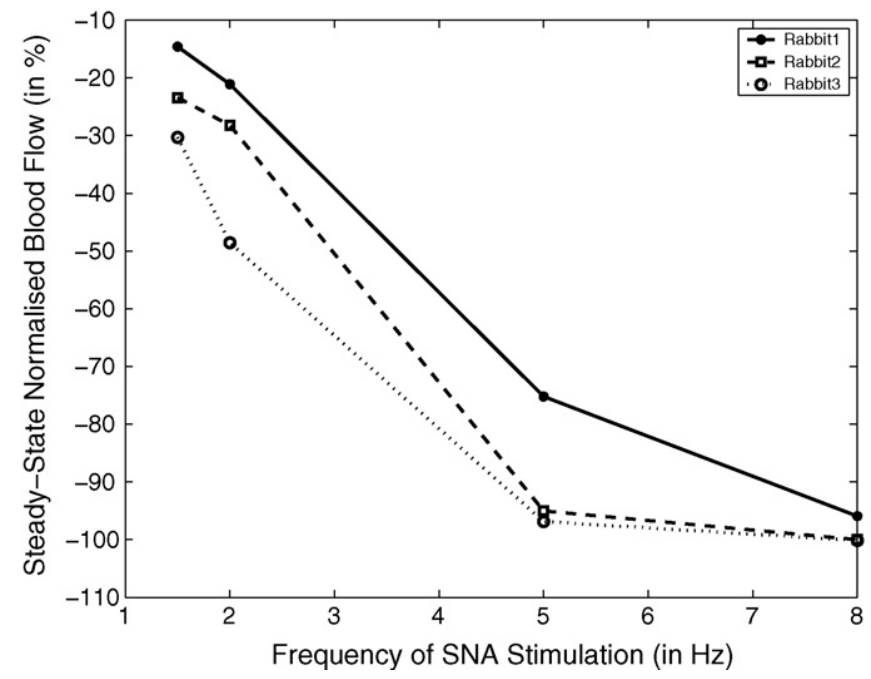

Fig. 9. 'Frequency' transform function for three animals.

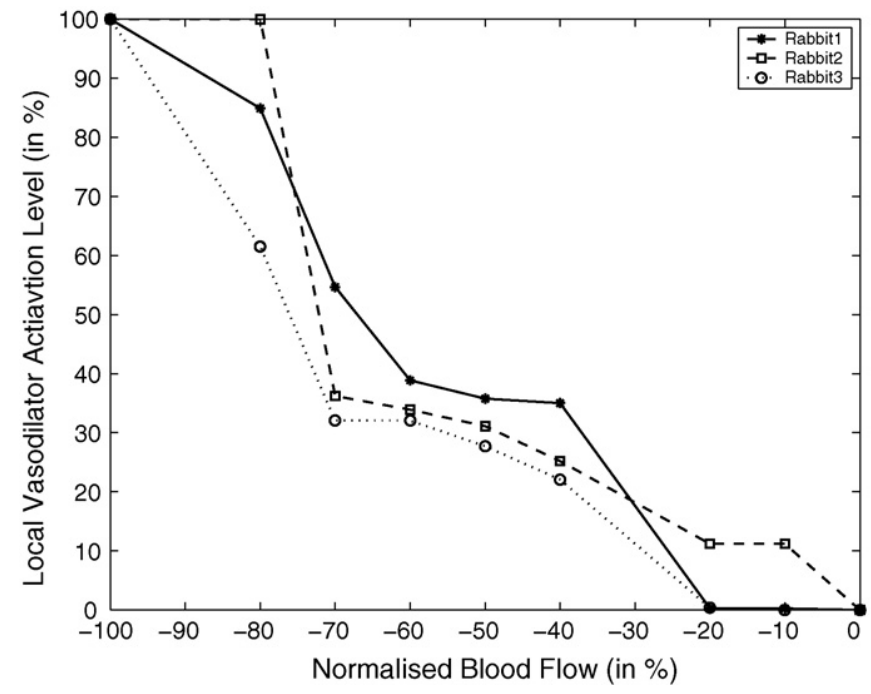

Fig. 10. Local vasodilators 'activation level' for the stimulation model.

The 'activation level' nonlinearity for the stimulation model of all three animals is shown in Fig. 10, while Fig. 11 shows the same nonlinearity for the recovery models. One distinctive feature of the models is the hysteresis effect which is returned in the identified 'activation level' characteristics. These roughly take the form shown in Fig. 12, which shows a classic hysteresis shape, typical of systems (e.g. electro-magnetic systems) which have 'memory' that takes some time to neutralise. In this case

Table 3

Dynamic block parameters for the stimulation and recovery models

\begin{tabular}{|c|c|c|c|c|c|c|c|}
\hline Animal set & Model & $k_{\mathrm{f}}$ & $k_{\mathrm{b}}$ & $\tau_{\mathrm{f}}(\mathrm{s})$ & $\tau_{\mathrm{b}}(\mathrm{s})$ & $J_{\text {tot }}$ & $J_{\text {test }}$ \\
\hline Rabbit 1 & Stimulation & 1.0639 & 40.6486 & 15.5304 & 33.9248 & 19.888 & 31.8497 \\
\hline Rabbit 1 & Recovery & 0.3058 & 524.568 & 30.7281 & 100.2366 & 65.9301 & 87.8868 \\
\hline Rabbit 2 & Stimulation & 1.3246 & 39.3004 & 15.2996 & 32.8461 & 67.0168 & 25.3646 \\
\hline Rabbit 2 & Recovery & 3.8658 & 506.7437 & 30.48 & 229.3788 & 542.9053 & 356.5051 \\
\hline Rabbit 3 & Stimulation & 1.3824 & 40.5726 & 15.5118 & 33.8387 & 152.7622 & 46.2158 \\
\hline Rabbit 3 & Recovery & 0.2318 & 1105.89 & 43.7512 & 211.528 & 1003.01 & 242.4465 \\
\hline
\end{tabular}




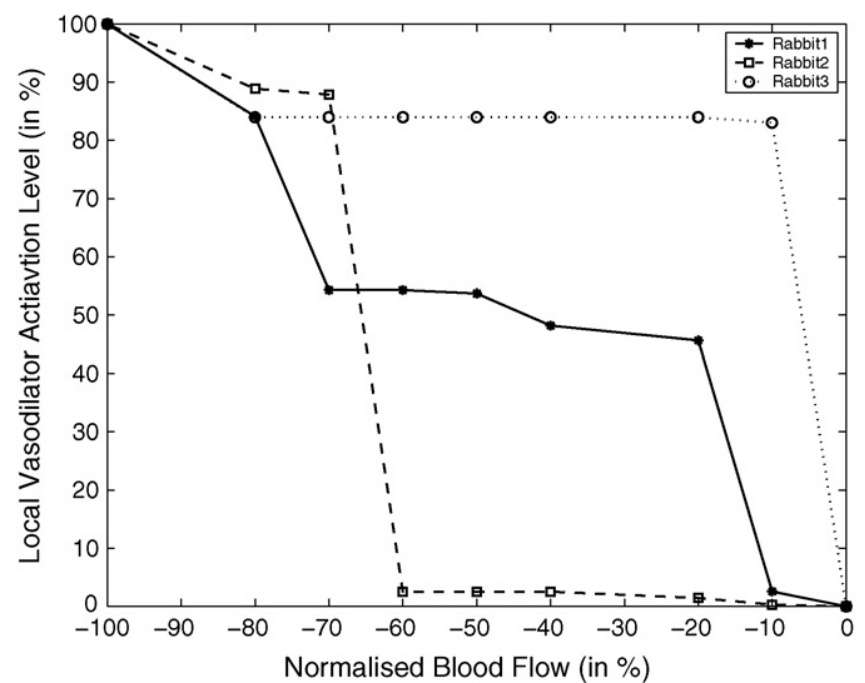

Fig. 11. Local vasodilators 'activation level' for the recovery model.

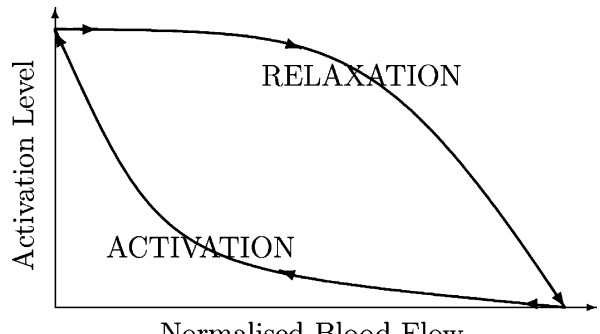

Fig. 12. Hysteresis effect.

the memory effect comes from the persistence of local vasodilators which take some time to be dispersed by the bloodstream.

A plot of results for Rabbit 1 is shown in Fig. 13, while the results for Rabbit 2 are shown in Fig. 14. There is a noticeable deterioration in the quality of the fit for the recovery stage of Rabbit 2 compared to Rabbit 1 . For brevity the model responses

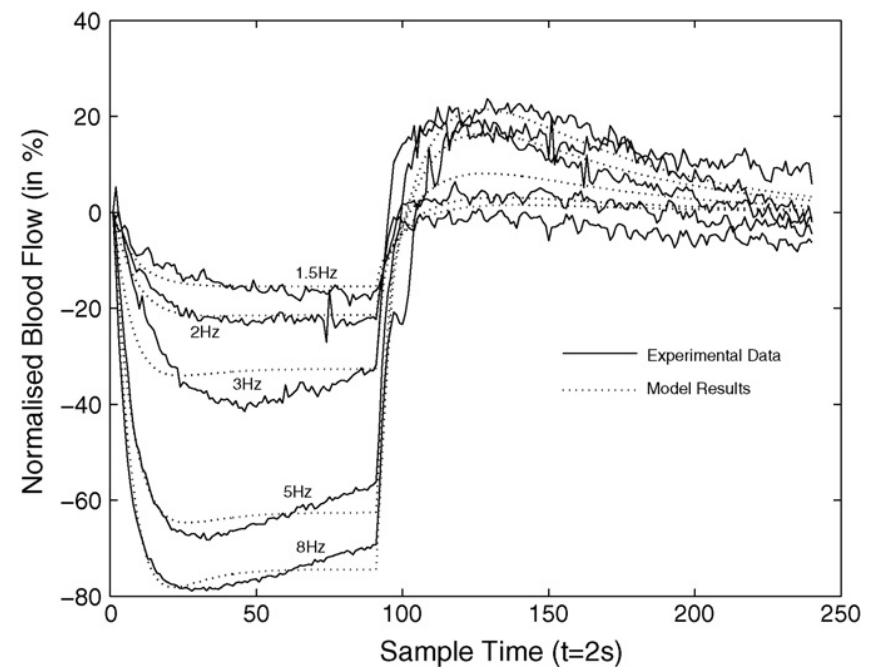

Fig. 13. Comparison of model output and experimental results for Rabbit 1 (stimulation and recovery together).

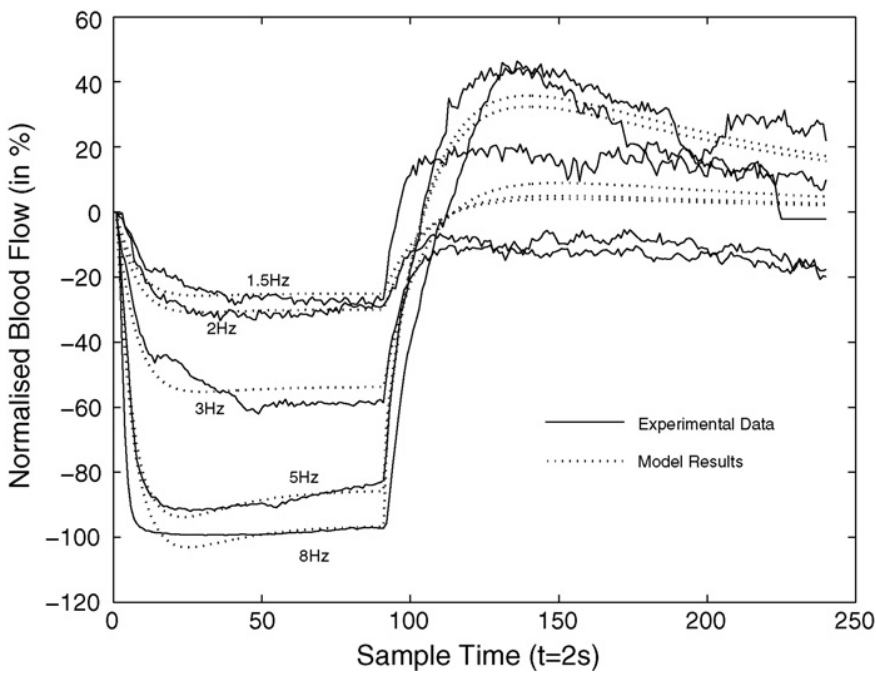

Fig. 14. Comparison of model output and experimental results for Rabbit 2 (stimulation and recovery together).

for Rabbit 3 is not presented graphically, however from the results in Table 3 it is evident that both the stimulation and recovery models severely under perform in the case of Rabbit 3 .

\section{Discussion}

This paper has presented a number of large-signal gray-box models for neurally induced vasoaction in the renal vasculature. The initially proposed model (Model A), even when manually optimised, provided evidence that there is some merit in this proposed model structure and gives some confidence that the model maps well onto the underlying physiology. However, the new alternative model (Model B) more accurately represents the local effector activation since it better reflects the relative spatial locations of systemic and local actions, leading to improved numerical results.

An improvement from previous work introduces separate optimisation of the model parameters for the stimulation and recovery stages. This technique yields mixed results for Model B. For Rabbit 1, noting that the cost (MSE) is a 'per sample' (mean) measure, the training MSE for the stimulation model is 19.9, while the recovery stage has a cost value of 65.9 (Table 3 ). Additionally, the test MSE for stimulation is 31.8 , while for recovery is 87.9 . These results suggest that the model structure is more representative of the vasculature response during neural stimulation and may need to be altered in order to better match the recovery stage.

This study, is advantageous over previous work by presenting model results for three different animals. Prior work concentrated on Rabbit 1 only, while in the current study, the stimulation and recovery models were optimised for two additional data sets. Additionally, in our previous studies, the models were trained on data for all five frequencies of stimulation. Here we have improved this technique by training the stimulation and recovery models on four frequencies of stimulation and testing them using the remaining frequency. A number of limitations of this study also need to be mentioned. 
As evident from the results, the selected model structure performs better for Rabbits 1 and 2 than it does for Rabbit 3 . Also, the results for individual animals are relatively good, but it has been difficult to obtain a true generic model. A number of possible explanations exist:

(1) Different voltage levels were used for each animal, as seen in Section 3.

(2) The various frequencies of stimulation were applied in a random order for each animal, resulting in different substances being active in each animal during the same stimulation frequency, inadvertently leading to differential response to the same input.

(3) There was no way to ensure that the same number of nerve fibers were recruited for each animal during stimulation.

The models presented in this paper focus on the renal vasculature only and caution must be exercised in any attempt to extend their applicability to other vasculature components or, indeed, to TPR in general. However, the renal vasculature is a major component of innervated resistance (approximately $30 \%$ ) and therefore the models have a significant relevance to TPR. In conclusion, the proposed model provides a good representation of the relationship between SNA and BF, and gives a good basis for developing a truly generic model. To date numerous research groups have focused on modelling the relationship between blood pressure (BP) and renal blood flow, where particular attention has been paid to autoregulation, including myogenic mechanisms and tubuloglomerular feedback (TGF) mechanism $[16,17]$. In this paper we consider the response of RBF to SNA stimulation. It is likely that autoregulation has a contribution to the total blood flow response here, however it is beyond the scope of this study to identify specific components and the magnitude of their action. Considering that blood pressure variations during one cycle of stimulation and recovery were very small (typical variations were $2-4 \mathrm{mmHg}$ ), it is conceivable that blood flow response was mostly due to SNA mediators and flow-sensing blood flow control mechanisms. It is also reasonable to suggest that the feedback loop in our model represents a sum of the responses of local vasodilatory and vasoconstrictory mechanisms.

A study on conscious animals [18], which focused on modelling BF response to SNA and BP, demonstrated that using both SNA and BP as inputs to the model leads to much better results than modelling separately the relationships between SNA and blood flow and that between BP and BF. Therefore, future work could attempt to incorporate previously identified models for renal blood flow response to blood pressure and nerve activity. This would be an important step forward, as it is suggested that $80 \%$ of the variation in renal blood flow can be accounted for by changes in blood pressure and SNA [18].

The proposed model structures deliberately separate dynamic and nonlinear elements (with a Hammerstein structure [19] in the feedback path) in an effort to make the model as transparent as possible and facilitate parameter tuning. Development of such models is particularly important in order to improve the understanding of the relationship between $\mathrm{RBF}$ and SNA, as changes in SNA seems to have a large effect on renal blood flow, as shown in this study, as well as in Ref. [18]. We also believe that a successful model can be helpful as part of the modelling effort to investigate the origins of low-frequency (circa $0.1 \mathrm{~Hz}$ in humans) oscillations in blood pressure. Current models utilise a relatively simple linear first-order dynamic element to represent the resistance component of the vasculature [20] and while this representation is adequate for smallsignal situations, it is known that oscillations of a significant amplitude can occur under certain physiological conditions, e.g. haemorrhage [21]. Inclusion of the counteractive vasodilatory mechanism in the models presented in this paper is likely to make a significant change to predictions of oscillation amplitudes (particularly at higher amplitudes) compared to current models utilising simple linear models.

\section{Acknowledgements}

This work was supported by the Irish Research Council for Science Engineering and Technology and NUI Maynooth.

\section{References}

[1] S. Guild, P. Austin, M. Navatikyan, J. Ringwood, S. Malpas, Dynamic relationship between sympathetic nerve activity and renal blood flow: a frequency domain approach, Am. J. Physiol. (Reg. Int. Comp. Physiol.) 281 (2001) R206-R212.

[2] G. DiBona, U. Kopp, Neural control of renal function, Physiol. Rev. 77 (1997) 75-197.

[3] C. Sorensen, P. Leyssac, O. Skott, N.-H. Holstein-Rathlou, Role of the rennin-angiotensin system in regulation and autoregulation of renal blood flow, Am. J. Physiol. (Reg. Int. Comp. Physiol.) 279 (2000) R1017R1024.

[4] R. Bellomo, J. Kellum, S. Wisniewski, M. Pinsky, Effects of norepinephrine on the renal vasculature in normal and endotoxemic dogs, Am. J. Respir. Crit. Care Med. 159 (1999) R1186-R1192.

[5] L.G. Navar, Integrating multiple paracrine regulators of renal microvascular dynamics, Am. J. Physiol. (Renal Physiol.) 274 (1998) F433-F444.

[6] C. Boulanger, P. Vanhoutte, The endothelium: a modulator of cardiovascular health and disease, Dialog. Cardiovasc. Med. 3 (1998) 4.

[7] D. Richardson, D. Randall, D. Speck, Cardiopulmonary System, 1st ed., Fence Creek Publishing, 1998.

[8] K. Tan, Y. Li, P. Gawthrop, A. Glidle, Evolutionary grey-box modelling for practical systems, Technical Report CSC-96019, 1997.

[9] V. Mangourova, J. Ringwood, Gray box modelling of arterial vasoaction, in: Proceedings of Irish Signals and Systems Conference, Dublin Institute of Technology, Dublin, Ireland, 2006.

[10] G. Eppel, K. Denton, S. Malpas, R. Evans, Nitric oxide in responses of regional kidney perfusion to renal nerve stimulation and renal ischaemia, Eur. J. Physiol. 447 (2003) 205-213.

[11] B. Leonard, R. Evans, M. Navakatikyan, S. Malpas, Differential neural control of intrarenal blood flow, Am. J. Physiol. (Reg. Int. Comp. Phys.) 297 (2000) R907-R916.

[12] M. Navakatikyan, B. Leonard, R. Evans, S. Malpas, Modeling the neural control of intrarenal blood flow, Clin. Exp. Pharmacol. Physiol. 27 (2000) 650-652.

[13] J. Ringwood, V. Mangourova, S. Guild, S. Malpas, A nonlinear model for vasoconstriction, in: Proceedings of the 6th IFAC Symposium on Modelling and Control in Biomedical Systems, Reims, France, 2006.

[14] R. Schoenberg, Optimization with the Quasi-Newton Method, Aptech Systems, Inc., Maple Valley, WA, 2001.

[15] J. diBona, L. Sawin, Frequency Response of the Renal Vasculature in Congestive Heart Failure, Circulation 107 (2003) 2159-2164. 
[16] N. Holstein-Rathlou, D. Marsh, A dynamic model of renal blood flow autoregulation, Bull. Math. Biol. 56 (1994) 411-429.

[17] R. Loutzenhiser, A. Bidani, L. Chilton, Renal myogenic response: kinetic attributes and physiological role, Circ. Res. 90 (2002) 1316-1324.

[18] C. Berger, S. Malpas, Modelling of the dynamic relationship between arterial pressure, renal sympathetic nerve activity and renal blood flow in conscious rabbits, J. Exp. Biol. 201 (1998) 3425-3430.
[19] L. Ljung, System Identification, Theory for the User, Prentice Hall PTR, 1999.

[20] J. Ringwood, S. Malpas, Control of renal blood flow-the case for a nonlinear model, Am. J. Physiol. 280 (2001) R1105-R1115.

[21] S. Malpas, D. Burgess, Renal SNA as the primary mediator of slow oscillations in blood pressure during haemorrhage, Am. J. Physiol. 279 (2000) R1299-R1306. 